\title{
MODELAGEM E SIMULAÇÃO DA TRANSFERÊNCIA DE CALOR NO ESCOAMENTO DE PASTAS DE CIMENTO EM COILED TUBING
}

\author{
L. P. FILHO ${ }^{1}$, C. E. G. PEREIRA ${ }^{1}$, E. C. H. PARAISO ${ }^{1}$, L. A. CALÇADA ${ }^{1}$ e C. M. \\ SCHEID $^{1}$ \\ ${ }^{1}$ Universidade Federal Rural do Rio de Janeiro, Departamento de Engenharia Química \\ E-mail para contato: calcada@ufrrj.br
}

\begin{abstract}
RESUMO - Flexitubos (coiled tubing) são utilizados em diversas operações tanto na construção quanto no abandono de poços de petróleo. No caso do fechamento do poço, esse tipo de tubo é utilizado para injeção de pasta de cimento na formação de tampões. Durante o escoamento da pasta, esta sofre variação de temperatura por causa do calor recebido devido ao atrito com a parede do tubo. Além disso, há uma troca térmica do tubo com o ambiente. Tais alterações podem influenciar no processo, modificando as propriedades físico-químicas da pasta e seu tempo de cura. Neste trabalho, foi desenvolvido um modelo matemático e implementado em linguagem FORTRAN na solução de um sistema de equações diferenciais parciais que descrevem as trocas térmicas entre os fluidos, a tubulação e o ambiente. A validação desse modelo foi feita utilizando dados de campo fornecidos por uma companhia exploradora de petróleo.
\end{abstract}

\section{INTRODUÇÃO}

O fechamento e abandono de um poço de petróleo é feito quando a exploração não é mais economicamente viável. Essa ação pode ser tomada também caso ocorra algum problema no poço que requeira fechamento. Esse processo pode ser realizado utilizando-se tampões mecânicos e/ou de cimento. Tampões são usados a fim de evitar a contaminação de aquíferos subterrâneos de água doce pela água da superfície, pela infiltração de petróleo ou gás ou pelas formações salinas (Shah e Lasat, 2003; NPC, 2011).

Como esse processo de fechamento e abandono de um poço não traz retorno financeiro, empresas tem buscado minimizar os custos sem que isto afete a segurança e eficácia da operação (Campbell e Smith, 2013). O uso do conjunto de coiled tubing (CT) trouxe grandes benefícios para esse tipo de operação por eliminar a necessidade de um equipamento de perfuração (drilling rig) e poder ser utilizado em outras etapas.

Apesar da eficácia dos flexitubos, o escoamento nestes pode gerar mudança nas propriedades físico-químicas das pastas de cimento devido ao aquecimento da pasta por causa do atrito com a parede do tubo, além de necessitar de uma maior pressão de bombeio. Sabendo disto, foi desenvolvido um modelo matemático a fim de prever as trocas térmicas envolvidas no escoamento de pastas de cimento quando os flexitubos estão enrolados, assim como, a mudança de temperatura que o fluido pode sofrer. 
Este modelo matemático foi implementado em linguagem Fortran, no Laboratório de Escoamento de Fluidos Giulio Massarani (LEF/UFRRJ). A partir de dados operacionais (entradas para o programa), serão gerados perfis de temperatura do tubo e do fluido ao longo tempo. Tais resultados serão comparados com dados de um estudo de caso obtidos de três tampões de cimento no fechamento de um poço de petróleo e, assim, verificando a eficiência dos modelos propostos e da utilização do software.

\section{REVISÃO BIBLIOGRÁFICA}

\subsection{Transferência de calor}

Foram feitos dois balanços de energia, um para o fluido e outro para o tubo. O fluido perde energia cinética, dissipada na forma de calor, devido ao atrito com as paredes do tubo. Sabendo disto, chega-se a equação do balanço energético onde foi considerado que a taxa de calor gerada pelo atrito é igual à taxa de calor necessária para que o fluido mude de temperatura mais o calor perdido por convecção interna (Equação 1).

$$
q_{\text {atrito }}=q_{\text {sensivel }}+q_{\text {conv. inter }}
$$

onde qatrito é a taxa de calor gerado por atrito (W), q qunsível é o calor sensível (W) e qconv inter é a taxa de transferência de calor por convecção interna (W).

Para o cálculo do calor gerado pelo atrito foi necessário verificar a perda de carga do escoamento na região do tubo enrolado no carretel.

\subsection{Perda de carga}

Como os fluidos reais apresentam certa viscosidade, o atrito destes com a parede de uma tubulação faz com que eles dissipem energia em forma de calor, gerando aquecimento do fluido. No escoamento de pastas de cimento, esse aquecimento do fluido pode provocar mudanças nas suas propriedades físico-químicas, além de poder alterar o tempo de cura delas, o que é prejudicial para a cimentação. Busca-se então prever a variação de temperatura sofrida pela pasta e a pressão à qual é submetida, mais próximas da situação do campo, para que essas condições sejam reproduzidas no laboratório e, assim, seja feita uma formulação da pasta adequada.

O cálculo da perda de carga total será calculado como um somatório das perdas de carga em cada camada e, caso na mesma camada haja alteração de diâmetro, esse somatório se abre nessa camada (Equação 2).

$$
\left(\frac{\Delta P}{\rho g}\right)=\left(2 f_{1} \frac{L_{1}}{D} \frac{v^{2}}{g}\right)_{1^{\underline{a}} \mathrm{c} c a m .}+\left(2 f_{2} \frac{L_{2}}{D} \frac{v^{2}}{g}\right)_{2^{\underline{a}} \mathrm{cam} .}+\cdots+\left(2 f_{n} \frac{L_{n}}{D} \frac{v^{2}}{g}\right)_{n \text {-ésima cam. }}
$$

onde g é a gravidade $\left(\mathrm{m} / \mathrm{s}^{2}\right), \Delta \mathrm{P}$ é a queda de pressão $(\mathrm{Pa}), \rho$ é a massa específica $\left(\mathrm{Kg} \cdot \mathrm{m}^{-3}\right), \mathrm{L}_{\mathrm{N}}$ é o comprimento de flexitubo na n-ésima camada $(\mathrm{m})$, v é a velocidade média $(\mathrm{m} / \mathrm{s})$ e $\mathrm{D}$ é o diâmetro do flexitubo (m). 


\subsection{Fator de atrito}

Estudos específicos de fator de atrito para escoamento de pasta de cimento foram desenvolvidos no LEF/UFRRJ e apresentados por Calçada et al. (2013). Nessas pesquisas, o escoamento da pasta era avaliado em trechos retos circular e anulares concêntricos. Para seções circulares, em espiral, Srinivasan et al. (1970) apud McCann e Islas (1996) apresentam uma correlação empírica para o cálculo do fator de atrito (Equação 3).

$$
f=\frac{0,084}{R e^{0,2}}\left(\frac{r}{R}\right)^{0,1}
$$

A partir da Equação 3, McCann e Islas (1996) deduziram a Equação 4 para fluidos não newtonianos, onde $a$ e $b$ são os parâmetros da equação e são dependentes do índice de comportamento $(n)$.

$$
f=\frac{1,06 a}{R e_{P}^{0,8 b}}\left(\frac{r}{R}\right)^{0,1}
$$

onde Re é o número de Reynolds (adim.), $\mathrm{Re}_{\mathrm{p}}$ é o número de Reynolds generalizado - modelo da potência (adim.) e n é o índice de comportamento - modelo da potência (adim.).

\subsection{Relação flexitubo-carretel}

A razão de curvatura para tubos espiralados é definida como a razão entre o raio interno do tubo e o raio de curvatura (Medjani e Shah, 2000). Para aplicação em espirais com mais de uma camada, a razão de curvatura deve ser diferenciada para a n-ésima volta, tendo em vista que o raio de curvatura leva em conta o raio do carretel $\left(R_{c}\right)$ e o raio externo do tubo de cada camada. A razão de curvatura pode ser definida a partir da Equação 5.

$$
\frac{r}{R}=\frac{r}{R_{C}+(2 . N-1) r}
$$

onde $\mathrm{N}$ é o número de camadas (adim.), $\mathrm{r}$ é o raio interno do flexitubo $(\mathrm{m}), \mathrm{R}_{\mathrm{c}}$ é o raio do carretel (m) e R o raio de curvatura $(\mathrm{m})$.

\section{METODOLOGIA}

Para calcular o perfil de temperatura, o calor sensível foi calculado ao longo do tempo e da posição, na direção axial, levando a equação diferencial apresentada na Equação 6. As condições inicial e de contorno usadas são apresentadas em (7) e (8), respectivamente.

$$
\begin{aligned}
& \rho_{f} c p_{f} \frac{\partial T_{f}}{\partial t}+\rho_{f} c p_{f} v_{z} \frac{\partial T_{f}}{\partial z}=E_{\text {atrito }}-h_{i} A_{i}\left(T_{f}-T_{m}\right) \\
& \text { C.I.: } \mathrm{t}=0 ; \mathrm{T}_{\mathrm{f}}=\mathrm{T}_{0} \\
& \text { C.C.: } \mathrm{z}=0 ; \mathrm{T}_{\mathrm{f} 0}=\mathrm{T}_{\mathrm{e}}
\end{aligned}
$$


onde $\rho_{\mathrm{f}}$ é a massa específica do fluido $\left(\mathrm{Kg} \cdot \mathrm{m}^{-3}\right), c_{p_{f}}$ é o calor específico do fluido (J/kg.K), $\mathrm{T}_{\mathrm{f}}$ é a temperatura do fluido $(K), T_{m}$ é a temperatura do metal $(K), v_{z}$ é a velocidade na direção $z$ $(\mathrm{m} / \mathrm{s}), A_{\mathrm{i}}$ é a área da superfície interna do flexitubo $\left(\mathrm{m}^{2}\right), \mathrm{h}_{\mathrm{i}}$ é o coeficiente convectivo de transferência de calor interno $\left(\mathrm{W} / \mathrm{m}^{2} \mathrm{~K}\right)$ e t é o tempo (s). O valor da temperatura utilizado na condição de contorno varia com o tipo de fluido que está sendo escoado. A água é alimentada na temperatura ambiente, enquanto que a pasta de cimento tem uma temperatura mais elevada devido ao processo de agitação e preparo nos tanques de mistura.

No balanço feito no fluido, a variação de temperatura considerada é a entre o fluido $\left(T_{f}\right)$ e o metal $\left(T_{m}\right)$. A energia perdida por atrito e recebida na forma de calor foi calculada utilizando-se a Equação 9.

$$
E_{\text {atrito }}=\Delta P_{\text {total }} Q
$$

No balanço de energia do tubo, considerou-se que o acúmulo de energia é igual aos fluxos de entrada e saída de energia através da parede, Equação 10. A condição inicial usada é apresentada em (11).

$$
\begin{aligned}
& \rho_{t} c p_{t} \frac{d T_{t}}{d t}=h_{i} A_{i}\left(T_{f}-T_{m}\right)-h_{e} A_{e}\left(T_{m}-T_{a m b}\right) \\
& \text { C.I.: } \mathrm{t}=0 ; \mathrm{T}_{\mathrm{t}}=\mathrm{T}_{\mathrm{amb}}
\end{aligned}
$$

onde $c_{p_{t}}$ é o calor específico do flexitubo (J/kg.K), Ae é a área da superfície externa do flexitubo $\left(\mathrm{m}^{2}\right), \mathrm{h}_{\mathrm{e}}$ é o coeficiente convectivo de transferência de calor externo $\left(\mathrm{W} / \mathrm{m}^{2} \mathrm{~K}\right)$ e $\mathrm{T}_{\mathrm{amb}}$ é a temperatura ambiente (K). Os valores das propriedades foram obtidos na literatura e os coeficientes convectivos de transferência de calor foram estimados no ajuste dos dados experimentais aos valores calculados pelo programa simulador.

Diferente do balanço de energia do fluido (Equação 6), não há o termo de derivada parcial quanto à posição para o balanço no flexitubo, visto que ele se encontra estático. Quanto ao fluxo de energia, considera-se a entrada na parede do flexitubo a convecção interna (diferença de temperatura entre o fluido e o metal) e, a saída, a convecção externa (diferença de temperatura entre o metal e o ambiente). Cabe ressaltar que a temperatura ambiente $\left(\mathrm{T}_{\mathrm{amb}}\right)$ aqui utilizada não é a temperatura do ar ( $\mathrm{T}_{\text {arr }}$. Para calcular a temperatura ambiente foi analisada a vizinhança de cada ponto (fração do tubo) compondo a temperatura de níveis de tubo com a temperatura ambiente.

A solução numérica das equações diferenciais parciais foi obtida discretizando o espaço por diferenças finitas e integrando no tempo utilizando a sub-rotina de domínio público LSODE. As equações foram programadas em linguagem FORTRAN.

\section{ESTUDO DE CASO}

Para a validação do programa desenvolvido, foi realizado um estudo de caso no fechamento de um poço com tampões de cimento e os dados foram fornecidos pela Petrobras. Três operações foram realizadas com o mesmo flexitubo para fechamento de um poço, onde foram feitos três tampões em diferentes profundidades por meio da cimentação. 
Em cada operação, para estabelecimento de cada tampão, um comprimento diferente de flexitubo está enrolado no carretel, já que os tampões são construídos em profundidades diferentes, sendo estas de 2140, 1990 e 204 metros para os tampões 1, 2 e 3, respectivamente. Ainda, cada tampão pode ter uma sequencia diferente de fluidos. Durante a operação, foram bombeados três fluidos, água, pasta de cimento e fluido de deslocamento.

Dados de temperatura no flexitubo foram coletados por meio de uma sonda de fibra ótica (DTS) presente no interior do flexitubo ao longo de todo o seu comprimento. Os dados eram coletados a cada meio metro e a cada minuto. A Figura 1 apresenta o resultado do perfil de temperatura durante a construção do Tampão 1.

Figura 1 - Dados de temperatura do registro geral da operação no carretel no Tampão 1 (Fonte: CENPES/Petrobras, 2015).

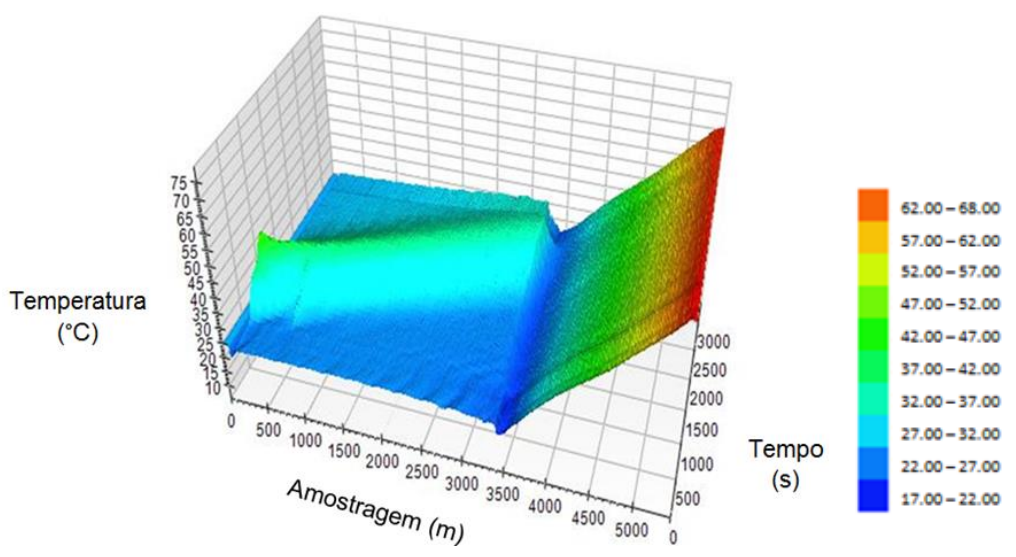

\section{RESULTADOS}

Os valores de temperatura do fluido ao longo do comprimento obtidos pelo programa foram comparados com os dados experimentais fornecidos no estudo de caso nos mesmos intervalos de tempo. Os gráficos gerados representam os intervalos onde a pasta de cimento estava sendo bombeada logo após água industrial em cada tampão (Figura 2).

Figura 2 - Dados calculados e experimentais de temperatura ao longo do comprimento e do tempo durante bombeio de pasta de cimento nos tampões 1,2 e 3 , respectivamente.
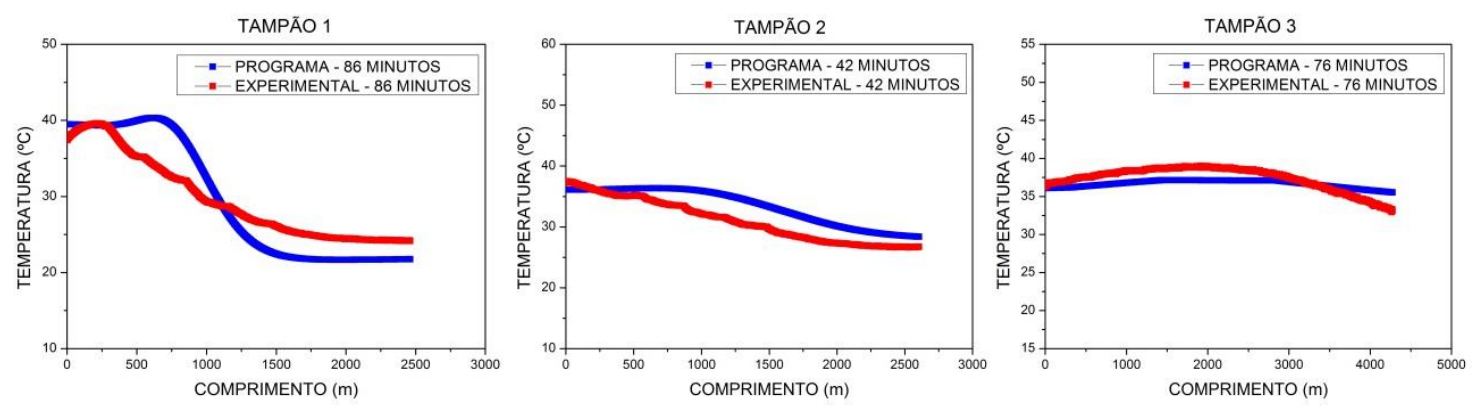
Na construção do Tampão 3, um maior comprimento de tubo estava enrolado, aumentando, assim, o contato tubo-tubo, e desta forma o modelo matemático, desenvolvido a partir de equações da literatura, apresenta maior proximidade com o experimental. Quando temos um menor comprimento de tubo enrolado (Tampões 1 e 2), a interação com o meio é maior, dificultando a análise matemática do problema, já que as variáveis do ambiente não são totalmente conhecidas. Contudo, como pode ser observado na Figura 2, o programa acompanha a variação de temperatura aferida experimentalmente. Tal comportamento pôde ser observado nas simulações feitas e o erro apresentado, em todos os casos, ficou abaixo de dez por cento.

\section{CONCLUSÃO}

O sistema de Equações Diferenciais Parciais foi resolvido por discretização do espaço e tempo possibilitando a obtenção do perfil de temperatura e pressão ao longo do comprimento do tubo e do tempo. Os coeficientes convectivos de transferência de calor foram estimados utilizando dados reais de campo fornecidos pela Petrobras. O perfil de temperatura estimado para intervalos de tempo nos quais fluidos foram bombeados em sequência foi satisfatório visto que as curvas experimentais e calculadas tiveram uma mesma tendência e desvio médio inferior a dez por cento. Desta forma, o programa simulador representou os dados experimentais de campo, possibilitando a utilização do mesmo para estimar a evolução da temperatura das pastas de cimento ao longo da posição e tempo.

\section{REFERÊNCIAS}

CAMPBELL, K.; SMITH, R. Permanent Well Abandonment. SPE, Tech 101, v. 9, n. 3, p. 25-27, 2013.

CALÇADA, L. A.; SCHEID, C. M.; PARAISO, E. C. H.; PEREIRA, C. E. G.; ROCHA, J. M. Pressure Drop in Cement Slurries Flow in Circular and Annular Regions in Primary Completion. BJPG, v. 7, n. 4, p. 129-139, 2013.

McCANN, R. C.; ISLAS, C. G. Frictional Pressure Loss During Turbulent Flow in Coiled Tubing. SPE/ICoTA, Texas, SPE 36345, fev.1996.

MEDJANI, B.; SHAH, S. N. A New Approach for Predicting Frictional Pressure Losses of Non-Newtonian Fluids in Coiled Tubing. SPE, Denver, Colorado, SPE 60319, mar. 2000.

NPC. Plugging and Abandonment of Oil and Gas Wells. NPC North American Resource Development Study, artigo \#2-25, 15 set. 2011. Disponível em: < https://www.npc.org/Prudent_Development-Topic_Papers/2-

25_Well_Plugging_and_Abandonment_Paper.pdf >. Acesso em: 14 nov. 2015.

SHAH, S.; LASAT, M. Development of an Environmentally Friendly and Economical Process for Plugging Abandoned Wells (Phase II). EPA, 2003. Disponível em: $<$ http://cfpub.epa.gov/ncer_abstracts/index.cfm/fuseaction/display.highlight/abstract/8743 /report/2003>. Acessado em: 25 nov. 2015. 